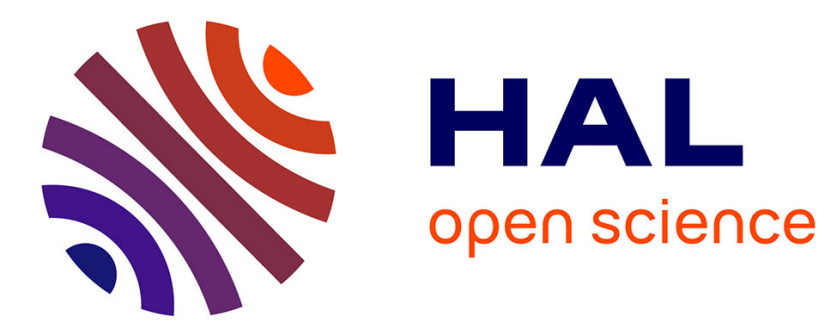

\title{
Swift Iodine ion modification of the structural and magnetotransport properties of $\mathrm{Fe} / \mathrm{Cr}$ systems
}

M. Kac, J. Zukrowski, M. Toulemonde, R. Kruk, V. Tokman, A. Polit, Y. Zabila, A. Dobrowolska, O. Synashenko, M. Marszalek

\section{- To cite this version:}

M. Kac, J. Zukrowski, M. Toulemonde, R. Kruk, V. Tokman, et al.. Swift Iodine ion modification of the structural and magnetotransport properties of $\mathrm{Fe} / \mathrm{Cr}$ systems. The Seventh International Symposium on Swift Heavy Ions in Matter, Jun 2008, Lyon, France. hal-00256380

\section{HAL Id: hal-00256380 \\ https://hal.science/hal-00256380}

Submitted on 14 Jul 2008

HAL is a multi-disciplinary open access archive for the deposit and dissemination of scientific research documents, whether they are published or not. The documents may come from teaching and research institutions in France or abroad, or from public or private research centers.
L'archive ouverte pluridisciplinaire HAL, est destinée au dépôt et à la diffusion de documents scientifiques de niveau recherche, publiés ou non, émanant des établissements d'enseignement et de recherche français ou étrangers, des laboratoires publics ou privés. 


\title{
Swift iodine ion modification of the structural and magnetotransport properties of $\mathrm{Fe} / \mathrm{Cr}$ systems
}

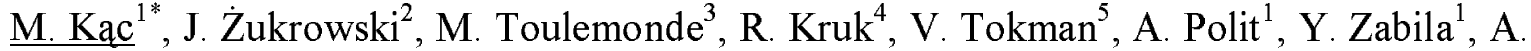 \\ Dobrowolska $^{1}$, O. Synashenko ${ }^{6}$, M. Marszałek ${ }^{1}$
}

${ }^{1}$ The Henryk Niewodniczański Institute of Nuclear Physics PAN, ul. Radzikowskiego 152, 31-342 Kraków, Poland

${ }^{2}$ Solid State Physics Department, Faculty of Physics and Applied Computer Science, University of Science and Technology AGH, al. Mickiewicza 30, 30-059 Kraków, Poland ${ }^{3}$ CIMAP-GANIL, ENSICAEN, Bd. H. Becquerel, BP 513314070 CAEN-cedex 05, France

${ }^{4}$ Institute of Nanotechnology, Forschungszentrum Karlsruhe GmbH, Postfach 3640, 76021 Karlsruhe, Germany

${ }^{5}$ Institute of Applied Physics, National Academy of Sciences of Ukraine, 58

Petropavlovskaya Str., Sumy, 40030, Ukraine

${ }^{6}$ Sumy State University, UA 40007 Sumy, R. -Corsakova, 2, Ukraine

PACS codes/keywords: $68.35 . \mathrm{Ct}, 75.70 .-\mathrm{i}, 76.80 .+\mathrm{y} / \mathrm{Fe} / \mathrm{Cr}$, multilayers, ion irradiation, interface roughness, magnetoresistance, antiferromagnetic coupling

* Corresponding author: e-mail Malgorzata.Kac@ifj.edu.pl, Phone: +004812 6628145, Fax: $+0048126628089$

Abstract
$\mathrm{Fe} / \mathrm{Cr} / \mathrm{Fe}$ trilayers and $(\mathrm{Fe} / \mathrm{Cr})_{20}$ multilayers prepared under ultrahigh vacuum conditions by thermal evaporation were irradiated with $200 \mathrm{MeV} \mathrm{I}^{13+}$ ions in the fluence range between $1 \times 10^{11}-8 \times 10^{12}$ ions $/ \mathrm{cm}^{2}$. The structural properties of the $\mathrm{Fe} / \mathrm{Cr} / \mathrm{Fe}$ trilayers and $(\mathrm{Fe} / \mathrm{Cr})_{20}$ multilayers were measured by $\mathrm{X}$ ray reflectivity $(\mathrm{XRR})$ and conversion electron Mössbauer Spectroscopy (CEMS). Magnetic exchange coupling between the Fe layers through the $\mathrm{Cr}$ spacer layer was observed by SQUID magnetization measurements. Magnetoresistance effect was measured using four probe method at room temperature.

The XRR spectra showed an increase of the interface roughness versus increasing irradiation fluence in the multilayers, while in the trilayers smoothening of the interfaces in the sample irradiated with fluence equal to $4 \times 10^{11} \mathrm{I} / \mathrm{cm}^{2}$ and very slight change for other fluences were observed. Improving of the interface structure in the trilayers at this fluence was observed also by CEMS. Moreover the Mössbauer spectra also confirm roughening of the interfaces as 
a function of fluence for multilayers. Before irradiation an antiferromagnetic coupling fraction dominated in all samples. After irradiation the changes of magnetic coupling were different in both types of samples. The trilayers were less sensitive to the irradiation fluence than multilayers and an increase of the antiferromagnetic fraction at small fluences was observed. In the multilayers a continuous decrease of the antiferromagnetic fraction as a function of fluence was evidenced. Vanishing of the antiferromagnetic coupling, observed for the largest fluence, resulted in the decrease of magnetoresistance effect in the $\mathrm{Fe} / \mathrm{Cr}$ multilayers.

\section{Introduction}

The multilayer systems like Fe/Cr, in which GMR (Giant Magnetoresistance) effect is observed, play an important role in magnetic storage devices. A proper functioning of magnetic multilayers depends strongly on the structural and magnetic properties of the materials. Reported experimental results describing the influence of the interface structure on the GMR value are inconsistent. Some of them show that GMR increases with the increasing interfacial roughness [1] contradicting other reports showing the opposite effect [2]. Nevertheless the correlation of GMR with the roughness is observed and is generally attributed to the variation of the interlayer coupling and in the spin dependent scattering at the interfaces [3]. Numerous papers concerning $\mathrm{Fe} / \mathrm{Cr}$ systems deal with multilayers, in which the contribution of many layers and interfaces involves final material properties. The $\mathrm{Fe} / \mathrm{Cr} / \mathrm{Fe}$ trilayers is an elementary system consisting of two ferromagnetic films coupled through the non-magnetic spacer. In such a simple system the number of layers and interfaces is reduced but the magnetic structure is preserved, so the analysis of trilayers can be useful for studying the structural and magnetic properties of more complicated multilayers systems. 
In this paper the studies of the magnetic and the structural properties of the $\mathrm{Fe} / \mathrm{Cr} / \mathrm{Fe}$ trilayers and $(\mathrm{Fe} / \mathrm{Cr})_{20}$ multilayers modified by swift heavy ion irradiation are presented.

\section{Experiment}

The $\mathrm{Fe} / \mathrm{Cr} / \mathrm{Fe}$ trilayers and $\mathrm{Fe} / \mathrm{Cr}$ multilayers were prepared on $\mathrm{Si}(100)$ substrates by thermal deposition in ultrahigh vacuum system with the base pressure of $10^{-8} \mathrm{~Pa}$. The Si single crystal covered with native $\mathrm{SiO}_{2}$ were ultrasonically cleaned and then heated during 1 hour at $700 \mathrm{~K}$ at pressure below $10^{-6} \mathrm{~Pa}$. The substrate purity was controlled by Auger Electron Spectroscopy (AES). During the deposition process the layer thickness and evaporation rate were controlled by a quartz microbalance. All samples were prepared at room temperature on $10 \mathrm{~nm}$ of $\mathrm{Cr}$ buffer layer and covered by $5 \mathrm{~nm}$ or $2 \mathrm{~nm} \mathrm{Cr}$ cap layer for trilayers and multilayers, respectively. In all samples the thickness of Fe layers was equal to 2 $\mathrm{nm}$ whereas the $\mathrm{Cr}$ spacer thickness was equal to $1.2 \mathrm{~nm}$ which corresponds to the first antiferromagnetic maximum in the exchange coupling between $\mathrm{Fe}$ layers through $\mathrm{Cr}$ layer [4]. The evaporation rates changed from $0.6 \mathrm{~nm} / \mathrm{min}$ to $1 \mathrm{~nm} / \mathrm{min}$ for $\mathrm{Cr}$ and $\mathrm{Fe}$, respectively. The measurements of structural, magnetic and magnetotransport properties were performed ex-situ. The sample structure was investigated by-X-Ray Reflectometry (XRR) using a Philips X'Pert MRD Pro diffractometer. $\mathrm{Cu} \mathrm{K}_{\alpha}$ radiation operated at $40 \mathrm{kV}$ and $30 \mathrm{~mA}$ was converted to a parallel beam by an X-ray lens with the aperture diameter of approximately 5 $\mathrm{mm}$. The size of the beam was restricted by crossed slits to $10 \mathrm{~mm} \times 5 \mathrm{~mm}$. All XRR measurements were performed in coplanar geometry with specular $\theta-2 \theta\left(Q_{z}\right)$ reflectivity scans. The XRR spectra were fitted using X'Pert Reflectivity software. Additionally the structure of layers and interfaces were analyzed basing on conversion electron Mössbauer spectroscopy (CEMS). All Mössbauer measurements were performed at room temperature with $100 \mathrm{mCi} \mathrm{Co}(\mathrm{Rh})$ source using $\mathrm{He}-10 \% \mathrm{CH}_{4}$ gas flow electron counter. The Mössbauer 
spectra were fitted using Voight-based method of Rancourt and Ping [5] as a sum of Gaussian components for the hyperfine magnetic field $\left(\mathrm{B}_{\mathrm{hf}}\right)$, isomer shift (IS) and quadrupole splitting (QS) distributions. The magnetic measurements were performed using SQUID magnetometer. Magnetoresistance was measured at room temperature using standard four probe technique in transversal geometry in an external field up to 1 Tesla. Irradiation experiments were performed at room temperature at the Vivitron in IReS in Strasbourg using $200 \mathrm{MeV} \mathrm{I}{ }^{13+}$ ions and the a fluence range from $1 \times 10^{11}$ ions $/ \mathrm{cm}^{2}$ to $8 \times 10^{11}$ ions $/ \mathrm{cm}^{2}$. The projected range of $200 \mathrm{MeV}$ I ions is equal to $7 \mu \mathrm{m}$ [TRIM-95], what is much bigger than total thickness of multilayers (about $80 \mathrm{~nm}$ ), therefore I ions were implanted in the substrate.

\section{Results and discussion}

The structure of $\mathrm{Fe} / \mathrm{Cr} / \mathrm{Fe}$ trilayers and $\mathrm{Fe} / \mathrm{Cr}$ multilayers modified by $200 \mathrm{MeV}$ iodine ion irradiation was analyzed by the CEMS and XRR studies. In figures $1 \mathrm{a}$ and $1 \mathrm{~b}$ Mössbauer spectra (with component fraction insets) measured for as - deposited (Fig. 1a) and irradiated with fluence $4 \times 10^{11} \mathrm{I} / \mathrm{cm}^{2}$ (Fig. 1b) trilayers are presented. Detailed analysis of components of the spectra was described in the previous paper [6]. The individual components correspond to the Fe sites with different number of $\mathrm{Cr}$ atoms which appear in the first or second coordination shell. Assuming very simple model one can estimate the roughness of the interfaces. $2 \mathrm{~nm}$ Fe layer ( $\sim 14$ atomic layers) with perfectly smooth interfaces should have three components: $33 \mathrm{~T}$ (bulk - like component in the middle of layer), $30 \mathrm{~T}$ (is the second $\mathrm{Fe}$ layer counting from $\mathrm{Cr}$ ) and $\sim 22 \mathrm{~T}$ (layer directly at the interfaces) with fractions equal to $70 \%, 15 \%$ and $15 \%$, respectively. As it is seen on the inset for as - deposited sample (Fig. 1a) the fraction of bulk like component is of about $55 \%$, the fractions of $30 \mathrm{~T}$ and $22 \mathrm{~T}$ components are a little bit larger than $15 \%$ and there is also the smallest field component which is related to the $\mathrm{Fe}$ atoms which diffused far into the $\mathrm{Cr}$ layer and have only $\mathrm{Cr}$ atoms 
in the first and second coordination shells. The component fractions differ from an ideal case with perfect, smooth interfaces; the real interfaces spread over 4 - 5 atomic layers taking into account about $5 \%$ of $\mathrm{Fe}$ atoms which diffused into Cr layers. After irradiation with fluence of $4 \times 10^{11}$ iodine ions $/ \mathrm{cm}^{2}$, increase of the bulk - like component fraction and decrease of smaller field component fractions were observed (Fig. 1b). It means that after irradiation with this fluence interfaces became smoother and the Fe atoms spread over 3 atomic layers at the interfaces. Larger value of the smallest field component as compared with as - deposited sample indicates that the Fe atoms diffused just a little into the $\mathrm{Cr}$ layers and still have some Fe atoms in close surroundings. In figures $1 \mathrm{c}$ and $1 \mathrm{~d}$ XRR spectra measured for as deposited (Fig. 1c) and irradiated with the same fluence $\left(4 \times 10^{11} \mathrm{I} / \mathrm{cm}^{2}\right)$ trilayers (Fig. 1d) were shown along with the fits. The spectra are very similar apart from the small shift of the maxima towards higher angles in the irradiated sample which can suggest decrease of the layer thickness. The fits confirmed the small decrease of layer thickness in irradiated sample verifying the decrease of interface roughness. Mean values of the interface roughness obtained from the fits were equal to $0.7 \div 0.8 \mathrm{~nm}(5-5.5$ atomic layers) for as - deposited and $0.4 \div 0.5 \mathrm{~nm}$ (3 atomic layers) for irradiated sample which very well corresponds to the results taken from the Mössbauer measurements and confirms smoothening of interfaces after irradiation with this fluence. The CEMS and XRR spectra measured for the trilayers irradiated with other fluences were very similar to the spectrum for as - deposited sample. The interface roughness estimated from the Mössbauer measurements and XRR fits is close to the values for the non irradiated sample.

In figure 2 the CEMS and XRR spectra for as - deposited and irradiated multilayers are presented. The Mössbauer spectrum taken for as - deposited multilayers (Fig. 2a) is similar to the spectrum of the as - deposited trilayers. A small decrease of bulk - like component fraction and increase of $23 \mathrm{~T}$ and $30 \mathrm{~T}$ component fractions indicates roughening 
of the interfaces as compared with the trilayers. This can be attributed to the accumulation of defects during deposition of subsequent $\mathrm{Fe}$ and $\mathrm{Cr}$ layers, therefore one can expect smoother interfaces at the beginning of multilayers and rougher at the end. After irradiation with the fluence $4 \times 10^{11} \mathrm{I} / \mathrm{cm}^{2}$ (Fig. 2b), the fractions of small field components increase while the fraction of bulk - like components decrease, which is an evidence of roughening of the interfaces. The Fe atoms spread over 6 atomic layers at interfaces in as - deposited sample and over 8 atomic layers at the interfaces in irradiated sample. In figures $2 \mathrm{c}$ and $2 \mathrm{~d}$ the XRR spectra measured for as - deposited and irradiated $\left(4 \times 10^{11} \mathrm{I} / \mathrm{cm}^{2}\right)$ multilayers are shown. In the pattern measured before irradiation Kiessig fringes and Bragg peak are seen. After irradiation fringes vanished in contrary to the Bragg peak which is well-resolved but shifted towards smaller angle. Vanishing of the fringes indicates that the interface roughness increased after irradiation. The different positions of the Bragg peaks (at $1.2^{\circ}$ and $1.4^{\circ}$ ) are assigned to two different bilayer periods. The Bragg peak at $1.4^{\circ}$ comes from nominal periodicity of the multilayers $(3.2 \mathrm{~nm})$, whereas the peak around $1.2^{\circ}$, with periodicity equal to $3.7 \mathrm{~nm}$, may correspond to the lattice with larger interface roughness (irradiated sample). Such superlattice should also appear for larger number of repetitions when the amount of defects increases. It means that the small angle peak should be visible also before irradiation. When we look at the as - deposited sample one can see slightly visible one slope of this peak hidden in the background. The intensity of this peak increases after irradiation but at the same time the intensity of the peak corresponding to the nominal periodicity decreases. Therefore after irradiation the interface roughnesses increased and caused the increase of the bilayer periodicity. Values of the interface roughness obtained from the fits are equal to $0.9 \mathrm{~nm}(\sim 6$ atomic layers) and $1.2 \mathrm{~nm}(\sim 8$ atomic layers $)$ for as - deposited and irradiated samples, respectively. As in the case of the trilayers interface roughness obtained from the XRR fits corresponds very well with the results of the CEMS measurements. In the case of multilayers 
the roughness on the $\mathrm{Cr}$ was about $60 \%$ smaller than the roughness on the Fe layers, which is in agreement with the known asymmetry of the $\mathrm{Fe} / \mathrm{Cr}$ and $\mathrm{Cr} / \mathrm{Fe}$ interfaces $[7,8,9]$. Basing on the CEMS and XRR spectra, the multilayers irradiated with other fluences can be divided into two groups: samples irradiated with small fluences are similar to as - deposited sample, and those irradiated with larger fluences are similar to the sample irradiated with fluence $4 \mathrm{x}$ $10^{11} \mathrm{I} / \mathrm{cm}^{2}$

The magnetic exchange coupling between the Fe layers through $\mathrm{Cr}$ spacer layer was observed in the trilayers and multilayers basing on the hysteresis loops obtained from SQUID magnetometer. In figure 3 exampled loops measured for the trilayers and multilayers are shown. The evolution of the shapes and values of remanence versus ion fluence was seen. In the trilayers at small fluences the remanence value is smaller than in as - deposited sample indicating the increase of the antiferromagnetic coupling fraction. For fluence equal to $4 \mathrm{x}$ $10^{11} \mathrm{I} / \mathrm{cm}^{2}$, for which smoothening of the interfaces was observed the fraction of the antiferromagnetic coupling is a little bit larger than for the as - deposited sample. At larger fluences the remanence value increased. In the multilayers with increasing irradiation fluence the curves became more square - the loops looked typical of ferromagnetic arrangement of magnetic layers and the remanence value increased reflecting a decrease of the antiferromagnetic coupling fraction. The fraction of the antiferromagnetic coupling calculated form hysteresis loops $\left(1-\left(\mathrm{R}_{\mathrm{m}} / \mathrm{M}_{\mathrm{sat}}\right)\right)$ for the tri- and multilayers versus irradiation fluence are shown in figure 4 . The changes of the magnetic coupling were different for the trilayers and multilayers. Trilayers are noticeably less sensitive to the irradiation as compared with multilayers in which, even for the smallest fluence, strong variation of the remanence was observed. Additionally in the trilayers non monotonic dependence with increasing ion fluence was seen. The fraction of an antiferromagnetic coupling increased for small fluences and reached 0 value of remanence $\left(1 \times 10^{11} \mathrm{I} / \mathrm{cm}^{2}\right)$ then for larger fluences decreased 
(remanence value increased). In the multilayers continuous decrease of the antiferromagnetic fraction as a function of fluence was observed. For the largest used fluence the fraction of antiferromagnetic coupling almost vanished in the multilayers, while in trilayers nearly $80 \%$ of sample still remained coupled antiferromagneticlly.

The giantmagnetoresistance, i.e. the maximum change of the resistivity related to resistance value at saturation field, is shown in figure 5. An initial decrease of magnetoresistance followed by a plateau and a further decrease of magnetoresistance with increasing irradiation fluence was observed. Moreover the variation of the shape of magnetoresistance curves is shown in insets of figure 5. After irradiation the curves became more sharped but are saturated at the same field. Such changes in the shape are observed when antiferromagnetic coupling gets broken. The decrease of magnetoresistance versus irradiation fluence did not reflect exactly the continuous decrease of antiferromagnetic fraction what can suggest that not only the magnetic coupling but also the interface structure has an influence on the GMR effect.

Calculations showing the influence of $200 \mathrm{MeV}$ iodine irradiation on the $\mathrm{Fe}$ and $\mathrm{Cr}$ layers using thermal spike model [10] (in equilibrium charge state) were performed. This model assumes that energy deposited by ions expands itself within the electron gas and it is transferred to the atomic lattice by electron-phonon interactions inducing an increase of lattice temperature. The ion track, defined as a cylindrical volume in which the melting temperature is surpassed, is calculated as a function of time. The calculation showed that 200 $\mathrm{MeV}$ I ions formed $2.5 \mathrm{~nm}$ track radius in Fe layers while in the $\mathrm{Cr}$ layer the melting temperature was not reached. Taking into account the maximal irradiation fluence used in the experiment and track radius created in $\mathrm{Fe}$, the estimation of the melting volume for the largest fluence was performed. This value is equal about $15 \%$, what means that during irradiation $15 \%$ of Fe layer was at liquid state at the same time the temperature around ion 
track in Cr layers was equal about $2070 \mathrm{~K}$ (melting temperature of $\mathrm{Cr}$ is equal $2148 \mathrm{~K}$ ). That can be one of the reasons for larger roughening on Fe layer. The calculation can be applied to the multilayers, but not for the trilayers for which equilibrium charge state is not reach [11]. It means that the irradiation conditions are not the same for the trilayers and multilayers because of differences in charge state which influence on electronic energy loss. Usually the electronic energy loss is proportional to the square of the incident charge state, and in the trilayers electronic energy loss is twice smaller as compared with multilayers. Therefore the energy deposited in the trilayers is smaller than in multilayers what can be dominant reason of smaller sensitivity of trilayers to ion irradiation.

Different behavior of the $\mathrm{Fe} / \mathrm{Cr} / \mathrm{Fe}$ trilayers and $(\mathrm{Fe} / \mathrm{Cr})_{20}$ multilayers observed under iodine ion irradiation was observed also for $\mathrm{Au}$ ion irradiations (in print) and can be also attributed to the various initial interface structure of the trilayers and multilayers. As it was shown basing on the CEMS and XRR measurements the structure of the $\mathrm{Fe} / \mathrm{Cr} / \mathrm{Fe}$ trilayers is better ordered than the structure of $\mathrm{Fe} / \mathrm{Cr}$ multilayers. Smoothening observed for the trilayers could be explained taking into account the limited solubility of bulk Fe and $\mathrm{Cr}$ materials and assuming similar limited solubility of thin films, which could cause the tendency of the Fe and $\mathrm{Cr}$ atoms for the segregation. The reason that the smoothening was only observed in the trilayers could their initially smoother interfaces and smaller electronic energy loss. The increase of interface roughness in the multilayers and appearance of a new lattice parameter, not corresponding to the first antiferromagnetic maximum, can be dominant cause for the strong magnetic and magnetotransport modification of the multilayers.

\section{Conclusions}

The effect of $200 \mathrm{MeV} \mathrm{I}^{13+}$ ion irradiation on the $\mathrm{Fe} / \mathrm{Cr} / \mathrm{Fe}$ trilayers and $(\mathrm{Fe} / \mathrm{Cr})_{20}$ multilayers has been studied. In the trilayers smoothening of the interfaces at fluence equal to 
$4 \times 10^{11} \mathrm{I} / \mathrm{cm}^{2}$ and very slight changes for other fluences were observed. The fraction of an antiferromagnetic coupling increased at small fluences and decreased at larger ones. In the multilayers increase of the interface roughnesses and of the layer thickness after irradiation with larger fluences was seen. The fraction of antiferromagnetic coupling and magnetoresistance effect decreased versus increasing irradiation fluence.

\section{Acknowledgements}

The authors express their thanks to F. Haas (IRES laboratory) and J.P. Stoquert (PHASE laboratory) for help with the ion experiment. M. Kac is grateful to European Commission through the Human Potential program Network EuNITT for their financial supports (Contract No. HPRN-CT-2000-00047). The work was partially supported by the National Network MANAR. The partial support of the Polish Ministry of Science and Higher Education with grant No N507 080 32/2310 is acknowledged.

\section{References:}

[1] E. Fullerton, D. M. Kelly, J. Guimpel, I. Schuller, and Y. Bruynseraede, Phys. Rev. Lett. 68 (1992) 859

[2] A. Paul, A. Gupta, S. M. Chaudhari, D. M. Phase, Vacuum 60 (2001) 401.

[3] A. Gupta, D. Kumar, Nucl. Instr. and Meth. In Phys. Res. B 244 (2006) 202

[4] M. Kac, M. Toulemonde, J. Jaworski, J. Juraszek, R. Kruk, S. Protsenko, V. Tokman, and M. Marszalek, Mol. Phys. Rep. 40, 89 (2004).

[5] D. G. Rancourt, J.Y. Ping, Nucl. Instrum. Methods B 58 (1991) 85

[6] M. Kac, M. Toulemonde, J. Jaworski, J. Juraszek, R. Kruk, S. Protsenko, V. Tokman, M. Marszałek, Vacuum, 78, 661 (2005).

[7] F. Kilnkhammer, Ch. Sauer, E. Tsymbal, S. Handschuh, Q. Leng, W. Zinn, J Magn. Magn. Mater. 161, 49 (1996).

[8] M. Kac, M. Toulemonde, J. Jaworski, J. Juraszek, R. Kruk, S. Protsenko, V. Tokman, M. Marszałek, Vacuum, 78, 661 (2005).

[9] M. Kubik, T. Slęzak, M. Przybylski, W. Karaś, J. Korecki, Vacuum 63(2001)337

[10] Z. G. Wang, Ch. Dufour, E. Paumier, and M. Toulemonde, J. Phys.: Condens. Matter, 6733 (1994) 6.

[11] M. Toulemonde, Nucl. Instrum. Methd. B 250(2006)263. 


\section{Figure legends:}

Fig. 1 .

CEMS spectra with corresponding $\mathrm{B}_{\mathrm{hf}}$ component fractions and XRR spectra measured for $\mathrm{Fe} / \mathrm{Cr} / \mathrm{Fe}$ trilayers before and after $200 \mathrm{MeV} \mathrm{I}$ ions irradiation with fluence equal to $4 \times 10^{11}$ ions $/ \mathrm{cm}^{2}$.

Fig. 2.

CEMS spectra with corresponding $\mathrm{B}_{\mathrm{hf}}$ component fractions and XRR spectra measured for $(\mathrm{Fe} / \mathrm{Cr})_{20}$ multilayers before and after $200 \mathrm{MeV} \mathrm{I}$ ions irradiation with fluence equal to $4 \mathrm{x}$ $10^{11}$ ions $/ \mathrm{cm}^{2}$.

Fig. 3.

Hysteresis loops measured by SQUID for $\mathrm{Fe} / \mathrm{Cr} / \mathrm{Fe}$ trilayers (left column) and $(\mathrm{Fe} / \mathrm{Cr})_{20}$ multilayers (right column) shown with increasing $200 \mathrm{MeV}$ I ions fluence: a) as - deposited, b) $2 \times 10^{11}$ ions $/ \mathrm{cm}^{2}$, c) $4 \times 10^{11}$ ions $/ \mathrm{cm}^{2}$.

Fig. 4.

Fraction of antiferromagnetic coupling for $\mathrm{Fe} / \mathrm{Cr} / \mathrm{Fe}$ trilayers and $(\mathrm{Fe} / \mathrm{Cr})_{20}$ multilayers as a function of ion fluence, determined from SQUID measurements. The broken line is a guide the eye.

Fig. 5.

Magnetoresistance (defined as a maximum change of the resistivity related to resistance value at saturation field) shown for $(\mathrm{Fe} / \mathrm{Cr})_{20}$ multilayers as a function of $200 \mathrm{MeV}$ I ions fluence. The broken line is a guide the eye 

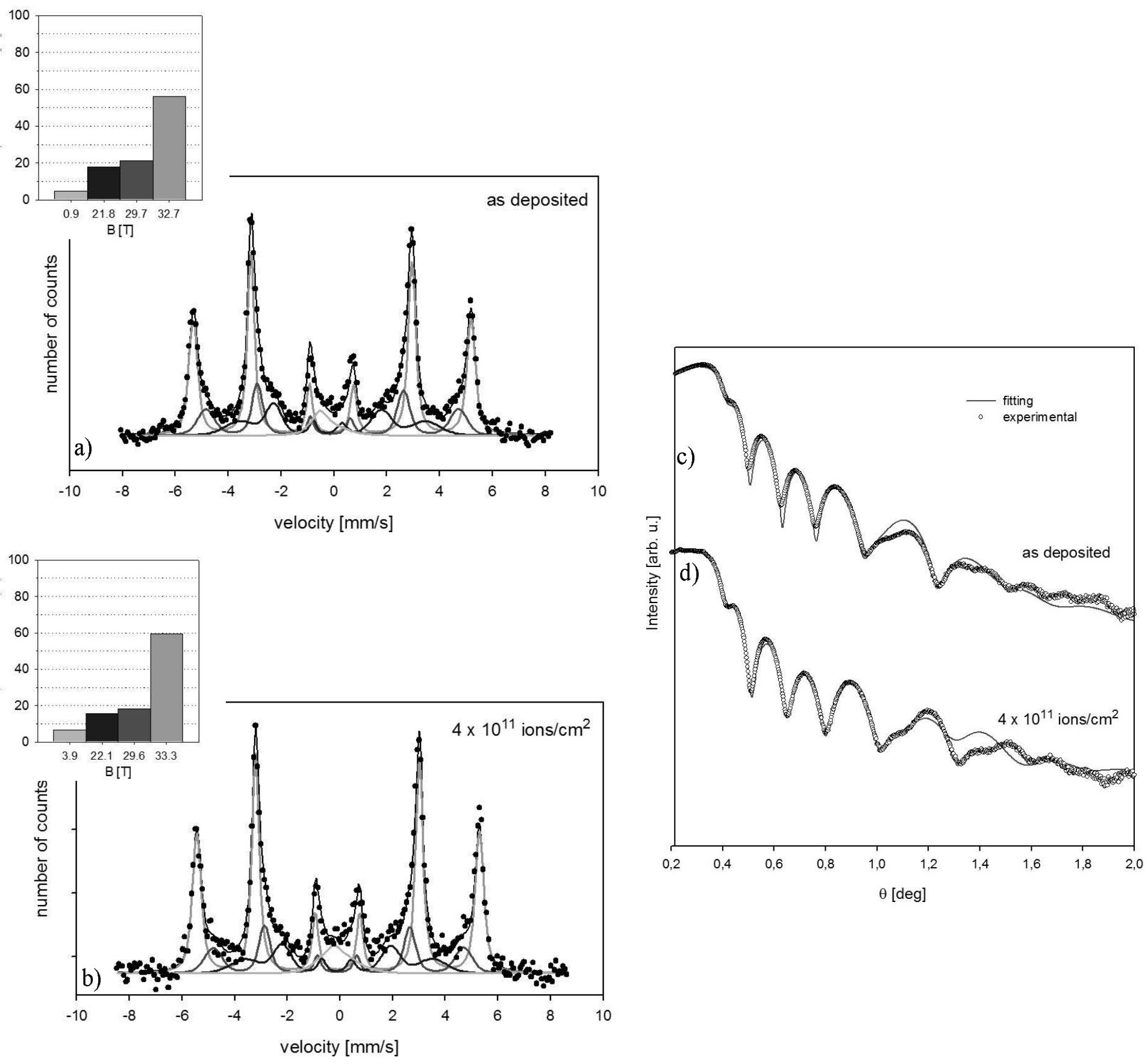

Fig. 1. 

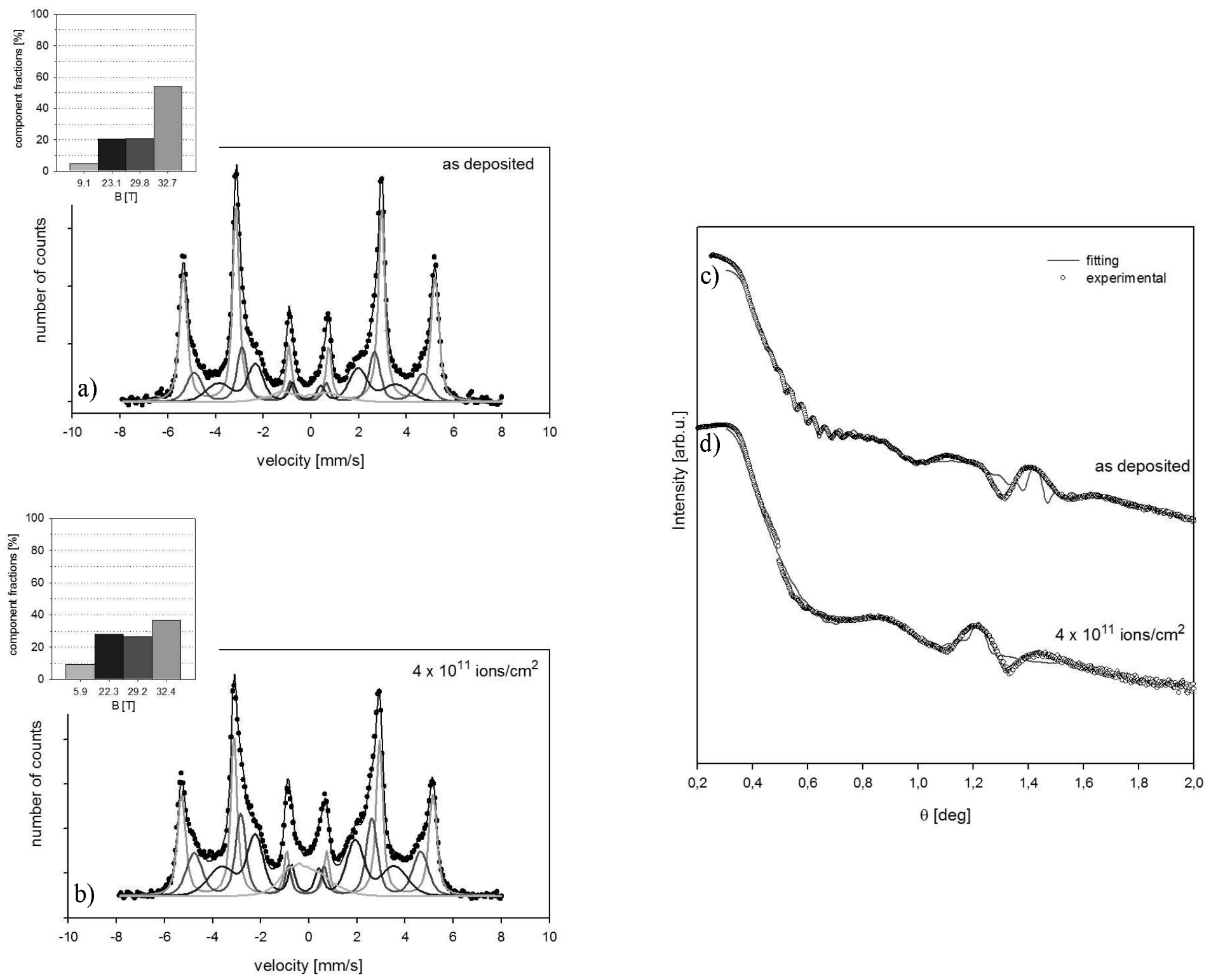

Fig. 2 . 
$\mathrm{Fe} / \mathrm{Cr} / \mathrm{Fe}$
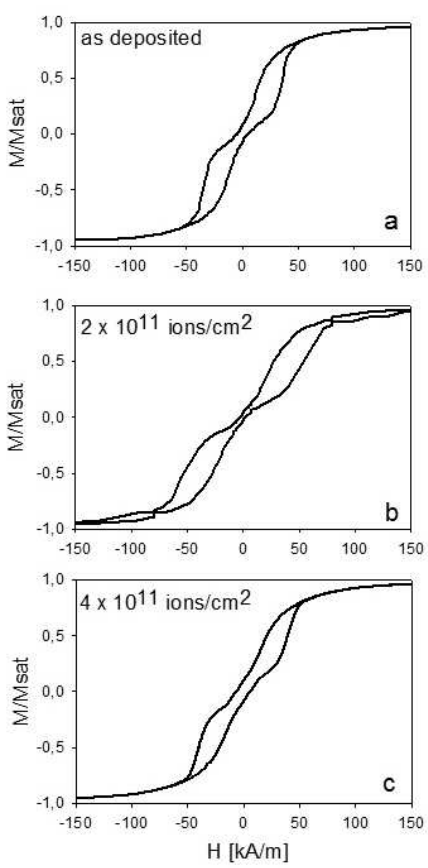

$(\mathrm{Fe} / \mathrm{Cr}) \times 20$
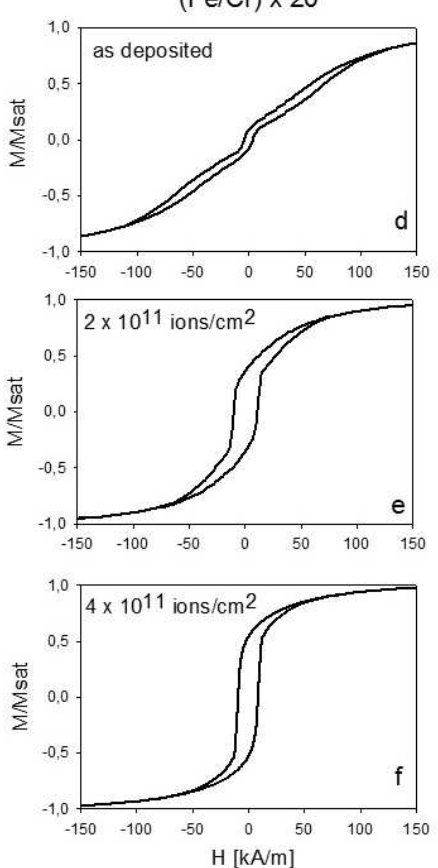

Fig. 3 . 


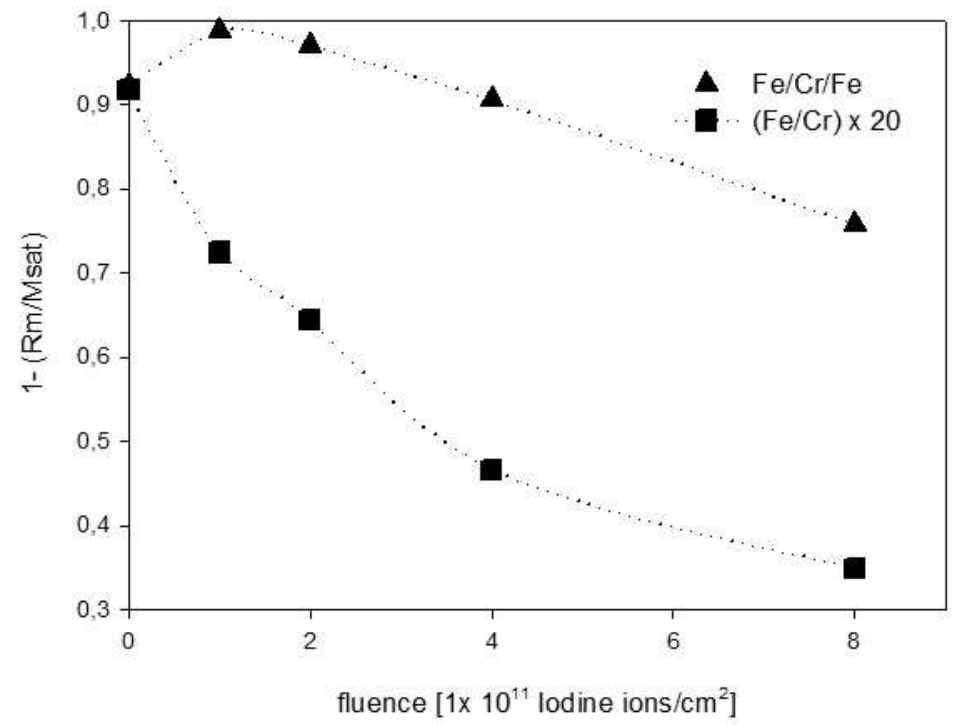

Fig. 4 . 


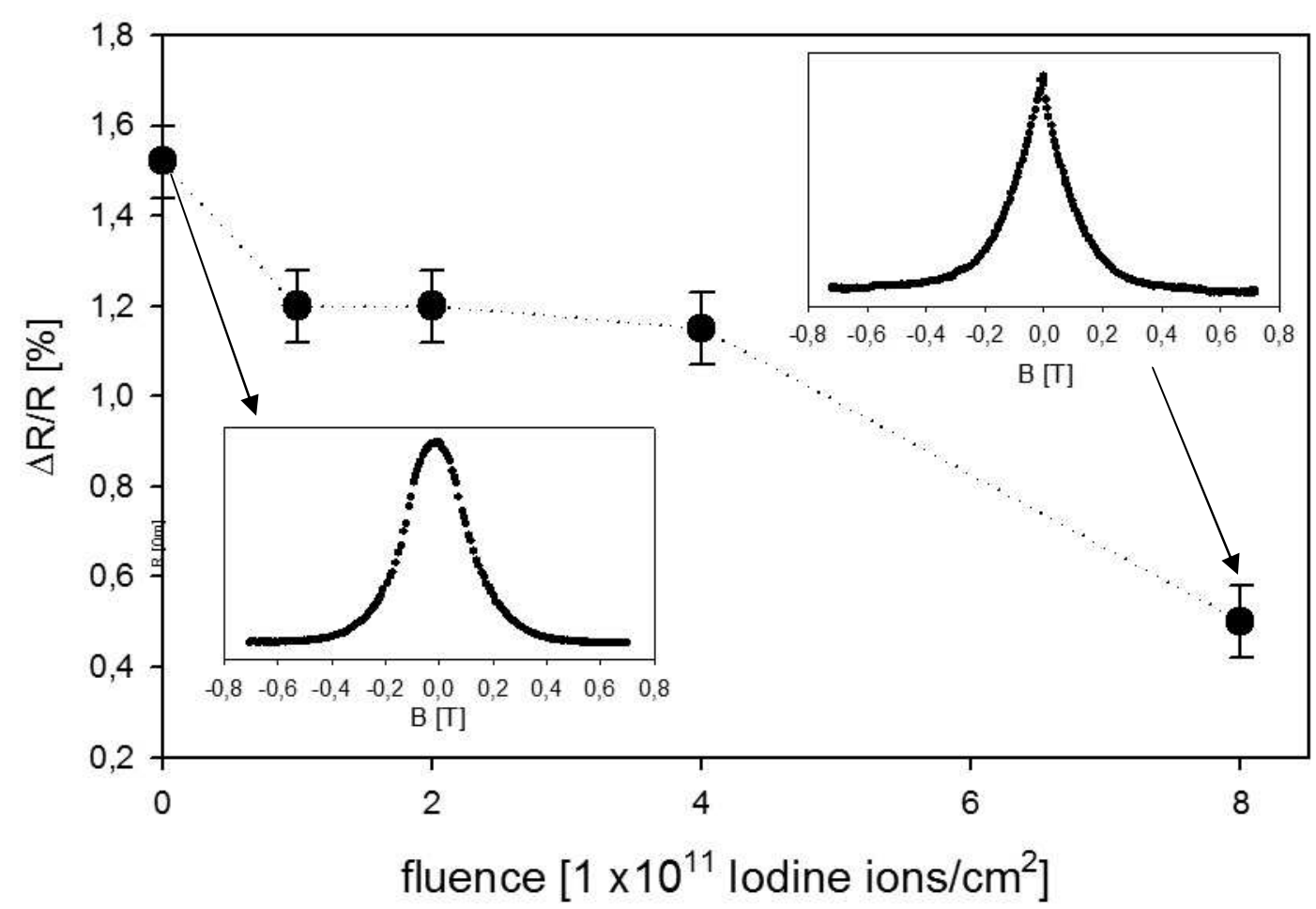

Fig. 5 . 